Sociologie et sociétés

\title{
Inclusion et exclusion à l'aune de la sociologie luhmannienne
} L'exemple de la pauvreté au Canada

\section{Stephen Schecter et Bernard Paquet}

Volume 32, numéro 2, automne 2000

Les promesses du cyberespace. Médiations, pratiques et pouvoirs à

l'heure de la communication électronique

URI : https://id.erudit.org/iderudit/001687ar

DOI : https://doi.org/10.7202/001687ar

Aller au sommaire du numéro

Éditeur(s)

Les Presses de l'Université de Montréal

ISSN

0038-030X (imprimé)

1492-1375 (numérique)

Découvrir la revue

Citer cet article

Schecter, S. \& Paquet, B. (2000). Inclusion et exclusion à l'aune de la sociologie luhmannienne : l'exemple de la pauvreté au Canada. Sociologie et sociétés, 32(2), 212-233. https://doi.org/10.7202/001687ar 


\section{$\Rightarrow$ \\ Inclusion et exclusion à l'aune de la sociologie luhmannienne:}

l'exemple de la pauvreté au Canada*

\section{stephen schecter}

Département de sociologie

Université du Québec à Montréa

C. P. 8888, Succ. Centre-Ville

Montréal (Québec), Canada, $\mathrm{H}_{3} \mathrm{C}$ 3P8

Courriel : schecter.stephen@uqam.ca

\section{bernard paquet}

Département de sociologie

Université du Québec à Montréal

C. P. 8888, Succ. Centre-Ville

Montréal (Québec), Canada, $\mathrm{H}_{3} \mathrm{C}$ 3P8

I'image h a bit uel I e de la société canadienne, comme d'ailleurs celle des autres sociétés dites avancées, postindustrielles, voire postmodernes, en est une caractérisée par un clivage grandissant, fruit d'un processus de globalisation qui nefait que masquer la tyrannie du marché. D'où, dans toute discussion autour de la pauvreté, la présupposition de départ que cetype de société produit de plus en plus de pauvres, qui portent aujourd'hui le stigmate del'exclusion, dont les dimensions sont à la fois multiples et cumulatives. Pourtant, les données statistiques sur la situation canadienne, comme celles des autres sociétés, démontrent souvent le contraire. Une lecture de ces données devrait en principe nous amener à conclure que la société contemporaine est marquée plutôt par une inclusion grandissante de la population et cela, peu importe la dimension sociale qu'on emploie pour la mesurer. Cette situation n'est pas si surprenante Iorsqu'on tient compte de la différenciation interne que Parsons (1977) a déjà signalée comme la caractéristique principale de la société moderne, dont les effets se voient dans la transformation du système de stratification. Aujourd'hui on témoigne d'une mobilité considérable et variée, qui s'étend au-delà de la sphère socioprofessionnelle pour couvrir celles du statut politique et d'identité sociale de tout acabit.

*Ce texte est le fruit d'une recherche sur la pauvreté au Canada subventionnée par le Conseil de recherches en sciences humaines du Canada. 


\section{l'exclusion sociale}

Depuis le début des années quatre-vingt, les phénomènes liés à la précarité sociale et à la pauvretéont étélargement redéfinis de part et d'autre del'Atlantique. La sociologie française a largement redéfini cette question à l'aune du concept d'exclusion sociale (Schnapper, 1981; Lion et M aclouf, 1982; Castel, 1992; 1994 ; 1995a; 1995b; Paugam, 1991; 1996 ; Paugam et al, 1993; D onzel ot, 1992 ; Fassin, 1996 ; et Gauthier, 1987 et 1995 pour le Q uébec). La sociologie américaine a plutôt réinterprété la question à l'aide du concept d'underclass (Auletta, 1982; Wilson, 1987, 1993; Katz, 1993). Les deux approches convergent cependant vers une analyse similaire de la pauvreté contemporaine. Centrales dans cette analyse sont les propositions suivantes. Les pauvres constituent un groupe qui se distingue par sa marginalisation vis-à-vis du reste de la société. Leur nombre augmente. Leurs désavantages sont cumulatifs. Ils sont négativement privilégiés selon l'ensemble des critères qui constituent le lot des citoyens moyens. Parmi les caractéristiques qui les marquent on trouvel'emploi instable sinon le chômage, la solitude affective, I'isolation sociale, la criminal ité, la maladie mentale, etc. La cristallisation parmi eux d'un ensemble desituations défavorables mène à la création d'un groupe distinct de plus en plus vulnérable, systématiquement exclu, une sorte de sous-classe dont la situation se détériore progressivement, signe d'une précarité qui risque de passer d'un processus à un état permanent. Et bien que l'analyse reste souvent ambiguë sur cette question, l'image projetée demeure celle d'un groupe social bien identifié par sa situation d'exclusion, la contrepartie et la conséquence d'une société néolibérale, ellemême caractérisée par l'inégalité croissante et l'émergence d'une société dualiste, scindée entre ceux qui ont et ceux qui n'ont pas accès aux ressources et aux expériences socialement valorisées. Vue de près cependant, l'analyse ne résiste pas à l'examen.

Les deux théorisations principales en France sont celles de Castel et de Paugam. Castel prétend que la pauvreté renvoie moins à un état qu'à un processus, processus décrit comme désaffiliation, marginalisation, ou précarité. La pauvreténous est présentée comme une situation de vulnérabilité qui, toutefois, s'apparenteà une «dynamique d'exclusion qui se manifeste déjà avant qu'elle ne produise ses effets complètement dé socialisants» (Castel, 1994, p. 11). Il distingue différentes étapes dans cette trajectoire, au bout de laquelle se trouve une «zone de désaffiliation » marquée par un «double décrochage : absence de travail et isolement relationnel ». (Castel, 1994, p. 13)

Cette façon de décrire la situation de la nouvelle pauvreté comporte cependant quelques désavantages majeurs. D'abord, traiter l'exclusion comme un processus permet aux adhérents à ce concept d'en parler en absence de toute verification statistique. On chercherait en vain dans les multiples écrits de Castel des chiffres pour appuyer ses affirmations sur l'exclusion. Ensuite, lorsque les chiffres révèlent que le nombre de personnes vivant dans une situation d'exclusion est beaucoup moindre que la théorie ne le suggère, on répond que l'exclusion n'est pas une situation mais un processus. Ce à quoi on peut dire que le concept devient tellement flou qu'il n'est guère utile pour saisir la nature de la pauvreté contemporaine. Et c'est d'autant plus le cas lorsque l'exclusion devient synonymeà la fois de la marginalisation et de la vulnérabilité, dont les 
dimensions couvrent autant les ressources financières et l'état de santé que les rapports intimes et la convivialité. Est-ce que les personnes seules sont seules par choix, par manque de vivres, par maladie mentale, ou par longévité? A-t-on les chiffres pour en déterminer la réponse ? Les cherchera-t-on lorsque l'exclusion fournit un concept couvre-tout?

Les analyses de Paugam ont le mérite de nous fournir des statistiques tout en insistant, elles aussi sur le fait que «les pauvres ne forment pas un ensemble social homogène» et quela pauvreté «est un phénomène multidimensionnel qui correspond aujourd'hui moins à un état qu'à un processus de disqualification sociale» (Paugam et al., 1993, p. 149). Dans ce rapport, Précaritéet risque d'exclusion en France, Paugam et al. ont examinéle lien entre la pauvreté économique et le statut d'emploi et, ensuite, celui entre la vulnérabilité sociale et le statut d'emploi. Ils concluent que la pauvreté tend à croître au fur et à mesure que l'emploi devient précaire, car seulement ceux dont on peut dire qu'ils détiennent un emploi stable non menacé ont une proportion de nonpauvres au-dessus de la moyenne nationale. M ais la catégorie d'emploi des chômeurs de deux ans et plus comprend elle aussi une majorité de non-pauvres. De plus, lorsqu'on examine leurs chiffres pour voir l'origine des pauvres, on découvre qu'une majorité de ceux classés comme pauvres ou très pauvres ont des emplois stables. Leurs propres chiffres sur la vulnérabilité sociale indiquent également que la moitié des chômeurs pendant plus de deux ans ne constitutent pas des citoyens vulnérables. Quand, par ailleurs, on analyse le statut d'emploi de ceux classés comme vulnérables, on trouve que presque les trois quarts détiennent des postes stables. (Paugam, et al., 1993)

Comment alors arrivent-ils à la conclusion que $20 \%$ de la population française vit une situation de précarité ou de fragilitétandis qu'un autre 20 à $30 \%$ se trouve dans une situation de «potentiellement fragiles malgrél'emploi stablequi leur garantit une forme d'intégration » (Paugam et al., 1993, p. 150) ? N'est-ce pas indicatif du caractère flou du concept de vulnérabilité, surtout lorsqu'il s'applique à un processus et non pas à une condition sociale ? Et n'est-ce pas précisément cette nébulosité qui leur permet de conclure que «le système actuel consacre la tendance vers un dualisme social institutionnalisé» (Paugam et al., 1993, p. 152) ? Pourtant, on ne voit aucune manifestation de cette tendance au niveau de l'action sociale, l'électorat français continuant à confirmer le divorce progressif entre le vote et les considérations socio-économiques observé depuis cinquante ans pour toutes les sociétés occidentales (Clark et Lipset, 1990).

À cela il faut ajouter d'autres considérations : l'association de facteurs différents, tels le statut matrimonial, le revenu familial, la santé, l'emploi et l'étendue des rapports sociaux, ne fournit pas en soi une idée de l'influence indépendanted'un facteur ni de la direction dans laquelle elle s'exerce. Trop souvent, comme Jencks l'a démontré dans sa discussion du concept del'underclass (1992) pour les États-Unis, on présume que I'un détermine l'autre tout en surestimant la signification de la corrélation. En l'absence d'études longitudinales de ces questions, il est difficile de conclure avec la certitude 
de Paugam et al. que la société actuelle tend vers un dualisme structurel. C'est un dilemme qui hantait la discussion de l'underclass de l'autre côté de l'Atlantique.

\section{l'underclass}

La paternitédu concept d'underdass revient non pas aux chercheurs en sciences sociales mais aux médias américains. En 1977, le magazine Time publiait un article portant le titre «TheAmerican Underclass» dans lequel il faisait état de l'émergence d'une nouvelle classe sociale dans les quartiers pauvres des grandes villes (cité par Katz, 1993). Par la suite, en 1981, un journaliste du New Yorker, Ken Auletta, publia une série de trois articles sur l'underclass qui prendront plus tard la forme d'une grande monographie (Auletta, 1982). Selon C. Jencks (1991), c'est à lui que l'on doit l'apparition de ce terme dans levocabulairecourant de la classe moyenne. Cen'est véritablement qu'en 1987, avec la parution de The Truly Disadvantaged deW. J. Wilson, que la première analyse scientifique prit forme. Plusieurs études portant sur l'underclass virent le jour depuis et, tout comme pour l'exclusion, la problématique de l'underclass stimule toujours nombre de discussions et de débats puisqu'il ne s'agit pas d'une notion univoque.

On peut cependant affirmer que ce concept suggère qu'il existe un groupe social distinct dans la sociétéaméricaine d'aujourd'hui qui ne bénéficie pas de ses avantages. Au contraire, il est proposé que ce groupe comprend un nombre disproportionné de délinquents juvéniles, de criminels récidivistes, de mères célibataires dépendantes de I'assistance sociale, de décrocheurs scolaires, de toxicomanes et de chômeurs. II habite les grands centres urbains, et surtout les ghettos noirs. Considéré par tous comme un fléau social, il est vu par certains comme la victime d'une société de plus en plus cruelle et inégalitaire, par d'autres commeun parasite dont l'irresponsabilité morale siphonne des ressources sociales. Commela plupart des observateurs prennent pour acquis l'existence de l'underclass, tout comme la plupart ailleurs adhèrent à la thèse de l'exclusion, les débats tournent autour des politiques à adopter ou de celles qui ne sont pas adoptées par des décideurs dont les intérêts se trouvent mieux servis autrement. II serait prudent cependant d'examiner la validité de la thèse de l'underclass avant de procéder à des conclusions d'ordre politique.

Christopher Jencks l'a fait dans son essai important «Is the American Underclass Growing ?», paru en 1991 et repris dans un livre portant sur la question un an plus tard (Jencks, 1992 ). Là, il démontre que les pauvres sont loin de constituer une catégorie homogène, et encore moins une classe. Une multitude de typologies de la pauvreté et des pauvres émergent de l'observation, et on comprend que la situation des pauvres s'améliore ou empire en fonction du type de pauvretédont ils relèvent. De plus, il apparaît que les politiques, tant celles de droite que celles de gauche, auxquelles les uns ou les autres imputent la responsabilité de cette situation sociale, n'y sont souvent pas pour grand-chose. Sans même parler de la quasi-impossibilité à identifier sérieusement et avec rigueur l'éventail des causes de la pauvreté.

Jencks montre, par exemple, que le taux de pauvreté a diminué aux États-Unis entre 1959 et 1974 pour augmenter légèrement par la suite. Pendant les années soixante- 
dix, environ un tiers des pauvres a échappéà la pauvretéau cours del'année qui a suivi la mesure, bien que certains y soient retombés par la suite. Pendant les années quatrevingt, en revanche, la pauvreté est devenue plus persistante, mais davantage en milieu urbain que rural. Quant aux chiffres relatifs à la transmision de la pauvreté ou du chômage chronique, c'est en vain qu'on les cherchera pour les États-Unis.

Croire quele chômage est synonyme de pauvreté constitue une erreur : plus de la moitié des hommes d'un âge compris entre 25 et 54 ans qui ne travaillent pas contre salaire vivent de nos jours dans les familles dont les revenus se situent au-dessus du seuil de la pauvreté. Plusieurs vivent dans des familles dont les revenus sont supérieurs au double du seuil de pauvreté. Un pour cent des hommes blancs et $4 \%$ des hommes noirs dans cette catégorie d'âge étaient pauvres sans emploi en 1969. Les chiffres correspondants pour la période 1985-1987 étaient respectivement de $2 \%$ et 6-8\%. Bien que le chômage chronique augmente les risques de devenir pauvre (il affecte différemment les blancs et les noirs), il est loin de constituer le seul facteur explicatif de la pauvreté. On ignore pourtant les causes précises de ces augmentations. Pour les blancs, par exemple, il est peu probable quel'augmentation constatée dans les taux de chômage chronique soit occasionnée par la pénurie d'emplois sur les marchés du travail. Mais il est pratiquement tout aussi certain qu'elle ne dérive en aucun cas d'une soi-disant culture de la paresse (ou d'une volonté plus ou moins délibérée de profiter du welfare), comme aiment à s'en gargariser les conservateurs, puisque le chômage chronique a continuéà augmenter pendant les années quantre-vingt, où l'on a pourtant exalté la répudiation de la culture permissive des années soixante et soixante-dix (Jencks, 1992). Cette mise en parallèle illustre la faiblesse, voire l'inconsistance des explications du chômage en termes de sous-cultures (de la pauvreté, de la paresse, du profit des revenus de remplacement, etc.).

Une vue tout à fait similaire se présenteautour dela question dela monoparentalité féminine. La proportion des mères célibataires qui bénéficiaient d'un subside social est passée de $29 \%$ en 1964 à 63\% en 1972, pour retomber à 45\% en 1988; la part des mères célibataires ayant un emploi demeurait constante pendant toutecette période. Quant à l'idée selon laquellel'underclass se caractérisait, entre autres, par une disproportion du nombre des mères célibataires, rien ne permet de le confirmer; et la seule chose dont on est à peu près sûr est quele «taux d'illégitimité» a augmenté dans toutes les strates de la société. $\mathrm{Ni}$ la prétendue largesse des subsides sociaux au non-emploi ni la pauvreté n'expliquent la monoparentalité. II est bien plus probable, en revanche, que les changements d'attitude en général face au mariage et à la conception hors mariage aient contribuéà la croissance des familles monoparentales; d'autant plus queces modifications s'articulent autour des mutations intervenues tant dans la situation économique des hommes et des femmes que dans les techniques de contraception. Avec l'augmentation du taux de divorce, augmente égal ement la proportion des mères célibataires, chez les femmes blanches comme chez les femmes noires, parmi les plus instruites comme parmi les moins instruites, même si le coût de ces changements est plus haut pour les femmes pauvres dans la mesure où elles ont moins de ressources à leur disposition (Jencks, 1992) . 
Le sens commun, de même qu'une certaine sociologie, a souvent présupposé que la pauvreté va généralement de pair avec un faible niveau de scolarité et que ce désavantage est hérité, contribuant ainsi à la reproduction des pauvres. Alors que pour le cas des États-Unis plusieurs auteurs en viennent à présumer une certaine corrélation positive entre pauvreté, appartenance à une minorité ethnique (généralement noire ou hispanique) et le double fait d'être peu instruit et d'avoir des parents peu instruits, le tout contribuant supposément à la constitution d'une underclass permanente, on constate en revanche que la scolarité des parents des jeunes noirs exerce une influence de plus en plus ténue sur le niveau de scolarité de leurs enfants. Entre 1972 et 1989, letaux de décrochage scolaire a chuté de manière plus marquée parmi les enfants dont les parents n'avaient pas fini l'école secondaire que parmi les enfants dont les parents avaient bénéficiéd'uneéducation plus élevée. Depuis 1950, les gains scolaires des enfants noirs de milieux défavorisés ont dépassé les gains des enfants provenant de milieux favorisés, blancs et noirs confondus (Jencks, 1992).

On affirme souvent par ailleurs que la pauvreté s'allie toujours plus intensément, en un mélange instable et explosif, avec une recrudescence de la violence urbaine. $M$ ais les chiffres récents indiquent le contraire : la violence a augmenté de façon significative (tant pour les Noirs que pour les Blancs) entre 1964 et 1974 et a décliné depuis 1980. Le taux d'homicide en 1989 était inférieur à ce qu'il était en 1970. Cela est d'autant plus intérressant que les chiffres sur la pauvreté ont suivi une courbe plutôt inverse, déclinant entre les années cinquante et la fin des années quatre-vingt pour augmenter quelque peu depuis. En outre, la convergence affirmée entre pauvreté et violence voile deux lacunes explicatives fondamentales, à savoir l'absence de données fiables relatives au rapport entre crimes violents et milieux socio-économiques des criminels et des victimes, d'une part, et, d'autre part, de données sur le rapport entre taux d'homicide et niveaux socio-économiques des quartiers où les homicides s'effectuent et des quartiers d'où proviennent les criminels et les victimes (Jencks, 1992).

II y a au moins deux conclusions à tirer de ces données. D'abord, la situation sociale des personnes au bas de la pyramide n'a pas systématiquement empiré depuis 1970. Les conditions économiques pour les travailleurs à faible formation auraient pu se détériorer, les familles monoparentales sont certes plus nombreuses aujourd'hui qu'il y a trois décennies, mais la dépendance des personnes vis-à-vis des revenus de remplacement alloués sous forme de subsides sociaux n'a pas augmenté depuis les années soixante-dix. Par ailleurs, l'analphabétisme, le nombre des mères adolescentes et la violence métropolitaine ont aussi reculé aux États-Unis. II serait ainsi illusoire de croire qu'il y a un groupe homogène au bas de l'échelle sociale qui partagerait toutes les caractéristiques généralement associées à la définition du milieu socialement et économiquement défavorisé, ou à la définition des exclus, des personnes en situation de fracture sociale.

Jencks a déjà démystifié bon nombre de conceptions courantes sur la nature des inégalités en général lors de son étude pionnière L'inégalité, parue en 1972. Là, il a montré que pour le système de stratification dans son ensemble, ni l'origine sociale, ni 
l'éducation, ni les connaissances, ni le milieu familial n'expliquent plus dela moitié de la réussitesocio-économique (Jencks et al., 1972). Letalent et le hasard y comptent pour autant, il suggère, cequi nous a obligés à réfléchir davantage sur la nature des inégalités contemporaines (Schecter, 1993). M ais comment pourrons-nous y réfléchir si nous demeurons tellement attachés aux formulations du problème que nous ignorons des chiffres qui les remettent en question?

Une étude canadienne récente qui ne touchait qu'indirectement la question de la pauvreté a néanmoins soulignéles écueils conceptuels et méthodologiques lors de nos discussions des questions concernant la distribution des inégalités. Dans leur article comparant des tendances au Canada et aux États-Unis, Wolfson et M urphy (1998) ont souligné l'importance de distinguer l'inégal ité de la polarisation, ce dernier concept mesurant plutôt ce qui se passe aux extrémités de la hiérarchie des revenus. Contrairement aux présuppositions courantes dans la presse et même dans les milieux universitaires, les auteurs ont trouvé, entre autres choses, qu'entre 1985 et 1995 la proportion de personnes ayant des revenus de classe moyenne aux États-Unis a augmenté et qu'eu égard aux revenus familaux, une fraction substantielle des familles canadiennes se trouvaient dans une meilleure situation (dans l'absolu) que leurs homologues américains en position similaire sur l'échelle de revenus (Wolfson et Murphy, 1998). De plus, au Canada, contrairement à la situation aux États-Unis, la position des plus pauvres s'est améliorée.

Dans lesillon de la discussion évoquée plus haut, nous allons procéder à une évaluation de la pauvreté au Canada en utilisant les mesures à notre disposition pour faire l'état de la situation. Ces mesures sont de nature statistique et concernent le revenu des personnes et des familles. Elles comportent certaines limites, mais au moins elles nous permettent d'avoir une idée de l'évolution de la pauvreté contemporaine dans le temps et de vérifier les hypothèses différentes à l'aune de quelques chiffres. Dans la mesure où l'on peut clarifier un certain nombre dequestions - par exemple, on peut voir, du moins, si la situation des pauvres s'est détériorée selon des critères strictement monétaires - , on pourrait peut-être mieux savoir de quoi on discute, bien que nous soupçonnions que le caractère politiquement chargé de ce débat soit lié au portrait général de la société que toute mise en question en termes de classes évoque. Nous y reviendrons, mais seulement vers la fin de ce texte. Nous pensons que l'analyse de la situation canadienne est particulièrement intéressante à cet égard, car elle est souvent considérée comme étant à mi-chemin entre les modèles américain et européen. Pourtant, les chiffres sur la pauvreté au Canada risquent non seulement de confirmer que les deux modèles analytiques développés ailleurs nes'appliquent pas à la situation canadienne, mais d'étayer la mise en question des thèses de l'exclusion et de l'underclass pour les sociétés où elles ont été élaborées.

\section{pauvreté et faible revenu}

Être pauvre signifie beaucoup de choses. C'est à la fois un état, une position sociale, une perception et un problème. Ainsi, on est pauvre par rapport à l'ensemble de la société, 
on se sent pauvre par rapport à celle-ci ou à un groupe social donné, on fait partie d'un groupe identifié comme pauvre, etc. Devant la multiplicité des définitions que I'on peut donner à la pauvreté, (H uman Resources Development Canada, 1998; Webber, 1998) il n'est pas étonnant de voir autant de manières de mesurer celle-ci. Une façon simple de sortir de cette impasse est d'utiliser un seuil de pauvreté.

Or, la multiplicité des seuils utilisés est souvent déconcertante. On peut cependant les regrouper en deux grandes catégories : les seuils normatifs ou absolus et les seuils re latifs. «Dans le premier cas, le seuil depauvreté est une mesure normative qui détermine ce qu'il en coûte pour survivre à un moment donné, dans une société donnée» (Langlois, 1987). On détermine ce seuil à partir d'un panier de base de biens et services sans lequel il serait impossible de survivre. Le second type de seuil est défini par rapport à la situation de l'ensemble de la société, il tient compte des biens normalement acquis par les autres (voiture, voyages, télévision, magnétoscope, etc.).

Mais ces définitions, ainsi que toutes les mesures choisies pour les rendre opérationnelles, sont trop souvent tributaires des résultats qu'on cherche à prouver. Celles utilisées par leConseil canadien du développement social (Ross et al., 1994), par exemple, risquent de diverger considérablement de celles utilisées par Sarlo (1996), commela figure 1 indique clairement. Devant cette panoplie de mesures, toutes liées à des considérations éthico-politiques, nous préférons utiliser celles de StatistiqueCanada.

Statistique Canada produit annuellement deux mesures de la faiblesse du revenu. II s'agit du Seuil de faible revenu ( $\mathrm{s} f \mathrm{r}$ ) et de la M esure de faible revenu ( $\mathrm{mf} r$ ). Toutes deux sont des mesures relatives car, à mesure que la société s'enrichit, le seuil augmente. C'est pourquoi de temps à autre la base à partir de laquelle le sf $r$ est calculé change, reflétant un changement dans le panier de biens et de services. Statistique Canada maintient, année après année, que ces deux mesures ne sont en aucun cas des

figure 1

Seuil de pauvreté pour une famille de quatre personnes vivant dans une grande ville, 1996

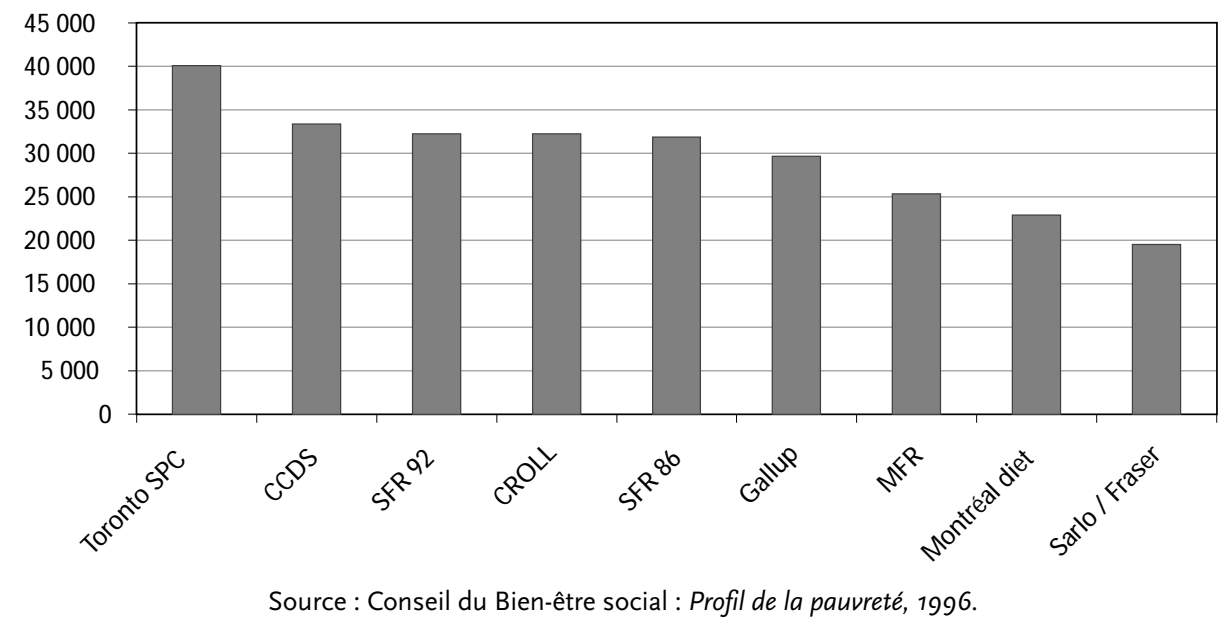


mesures de la pauvreté(Fellegi, 1998), mais mal grétout elles sont utilisées par de nombreux organismes de cette manière. Nous les suivrons, tout en consi dérant ces mesures plutôt comme des indicateurs de pauvreté, parce qu'elles nous permettent de voir comment ces indices évoluent dans le temps. Dans cette perpective, c'est plus le sens des variations qui nous intéresse que les nombres absolus que fournissent ces mesures.

La mesure la plus couramment utilisée, le sf $r$, peut être décrite comme étant le niveau de revenu où une famille dépense plus que la famille moyenne pour se loger, se nourrir et se vêtir. À I'origine, on a établi qu'une famille moyenne dépensait $50 \%$ deson revenu pour les besoins de base. On a ajouté $20 \%$ (de manière tout à fait arbitraire) à ce chiffre pour en arriver à l'idée qu'une famillequi dépensait $70 \%$ de son revenu était dans une position peu enviable. Statistique Canada a alors converti ce $70 \%$ en un ensemble de seuils ventilés selon la taille de la famille et la grandeur de la région de résidence.

Or, depuis l'introduction en 1969 de cette mesure, les dépenses moyennes pour les besoins de base sont passées de $50 \%$ à $35 \%$. On a donc révisé quatre fois cette mesure depuis son entrée en vigueur. Le plus récent seuil est fondé sur la base de 1992. En plus de cette révision périodique, les seuils sont ajustés chaque année au coût de la vie (Indice des prix à la consommation ou ipc). Plus récemment, Statistique Canada calcule des sf $r$ pour les revenus avant et après impôt, marquant ainsi le fait qu'en 1969, l'impôt était davantage une dépense et qu'aujourd'hui il est un important mécanisme de redistribution des revenus.

La seconde mesure qu'offre Statistique Canada est relativement plus simple. La mesure de faible revenu ( $\mathrm{mf} r$ ) est fixée (de manière encore tout à fait arbitrai re) à $50 \%$ du revenu médian ajusté selon la taille et la composition dela famille. Ainsi, le premier membre de la famille a un poids de 1 et le second, quel que soit son âge, a un poids de 0,4 . Les membres suivants ont un poids de 0,4 s'ils ont 16 ans ou plus et 0,3 s'ils ont moins de 16 ans. Toutefois, la $\mathrm{mf} r$ ne tient pas compte de la taille de l'agglomération.

Toutes ces considérations prises en compte, nous procéderons à l'examen de la pauvretéau Canada en faisant une analyse en séries chronologiques de ses mesures de faible revenu sel on certaines catégories.

\section{quelques statistiques sur le faible revenu au canada}

La proportion de personnes à faible revenu au Canada a décliné significativement depuis 1970, et ce, peu importe la mesure employée. Le pourcentage de personnes seules classifiées comme étant à faible revenu selon le barème du seuil de faible revenu est passé de $42,8 \%$ en 1969 à $26,8 \%$ en 1980 . Pour les familles, la réduction était plus frappante encore : passant de $20,8 \%$ en 1969 à $9,3 \%$ en 1980 . Depuis, il y a eu une légère augmentation dans le pourcentage de familles pauvres, mais la situation de personnes seules continuait de s'améliorer. Si on utilise la mesure du faible revenu et non pas le barème du seuil de faible revenu, on enregistre un déclin dans le pourcentage de personnes à fai ble revenu depuis 1980 pour toutes les catégories (StatistiqueCanada, cat. : 13-207-xpb ; 13-551-xpb ; 13-569-xpb ; 13-582-xpb). 
figure 2

Proportion de familles et de personnes seules à faible revenu (SFR Base 1992), 1980 à 1996.

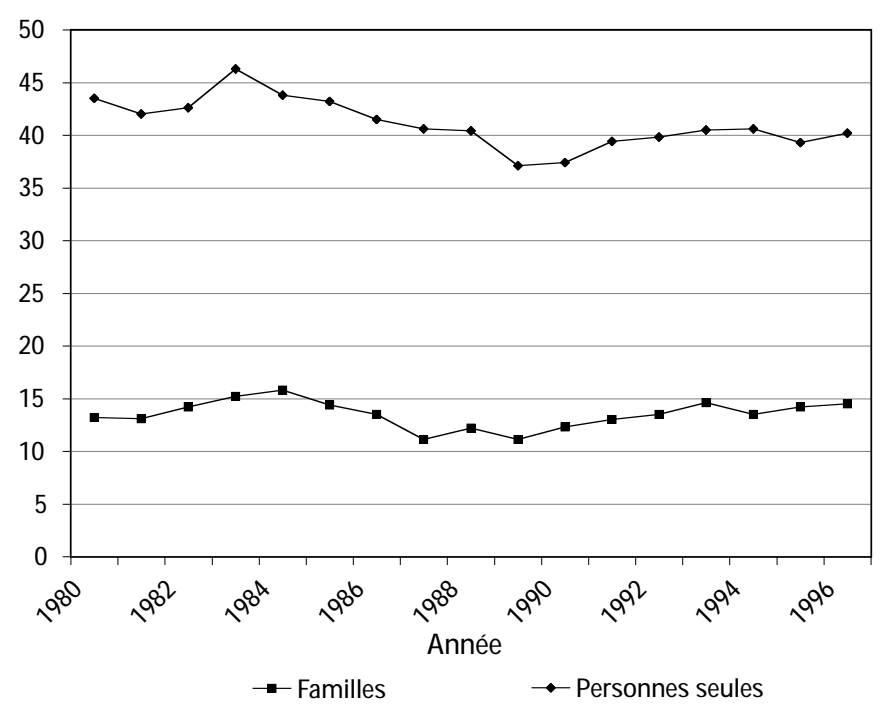

Source : Statistique Canada, cat. : 13-551-XPB et 13-569-XPB.

figure 3

Proportion de familles et de personnes seules à faible revenu (Mesure de faible revenu), 1980 à 1996.

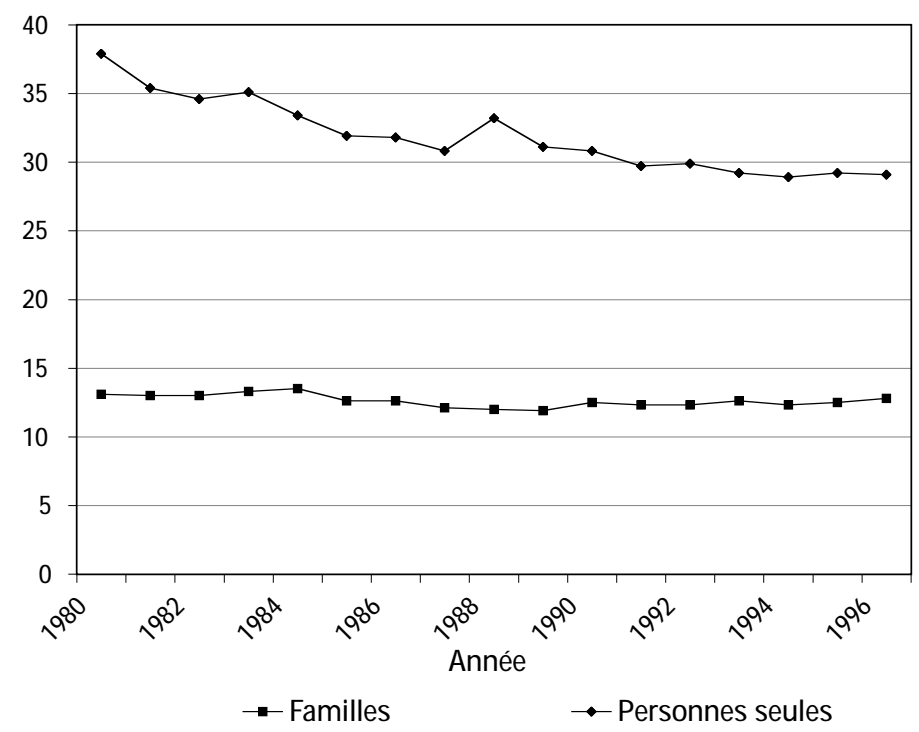

Source : Statistique Canada, cat. : 13-582-XPB. 
figure 4

Proportion de personnes à faible revenu avant et après impôt

(SFR base 1992), 1980 à 1996.

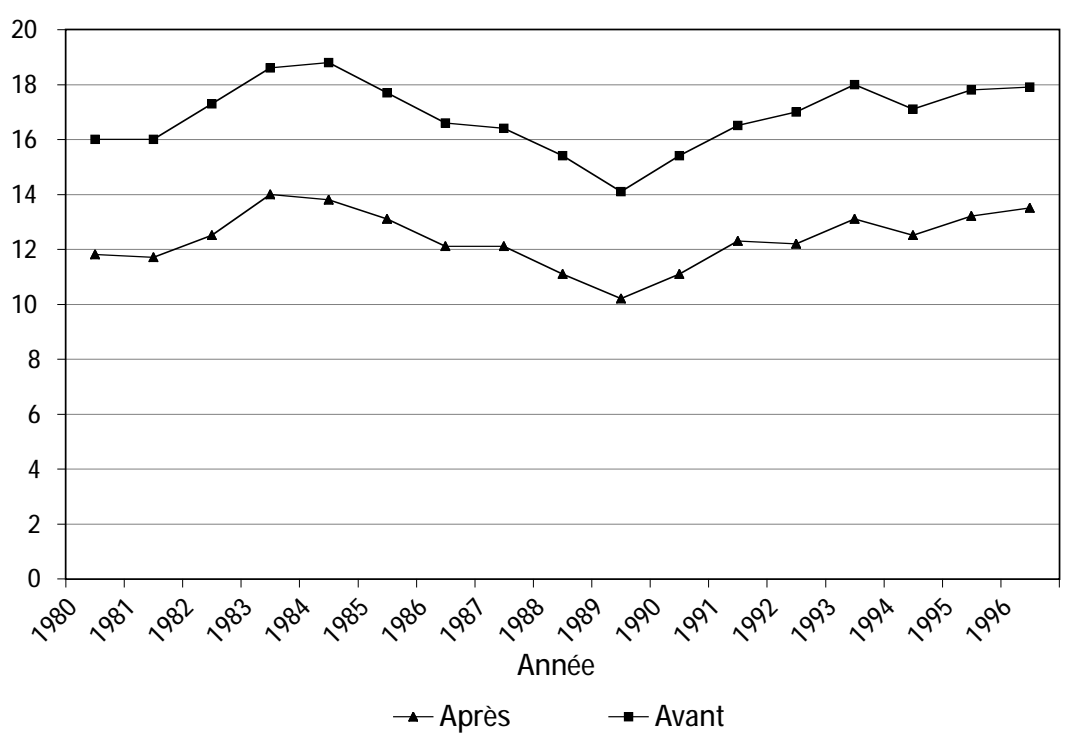

Source : Statistique Canada, cat. : 13-592-XPB et 13-551-XPB.

Figure 5

Coefficients de GINI selon différents concepts de revenu pour les personnes seules, Canada, 1971 à 1995

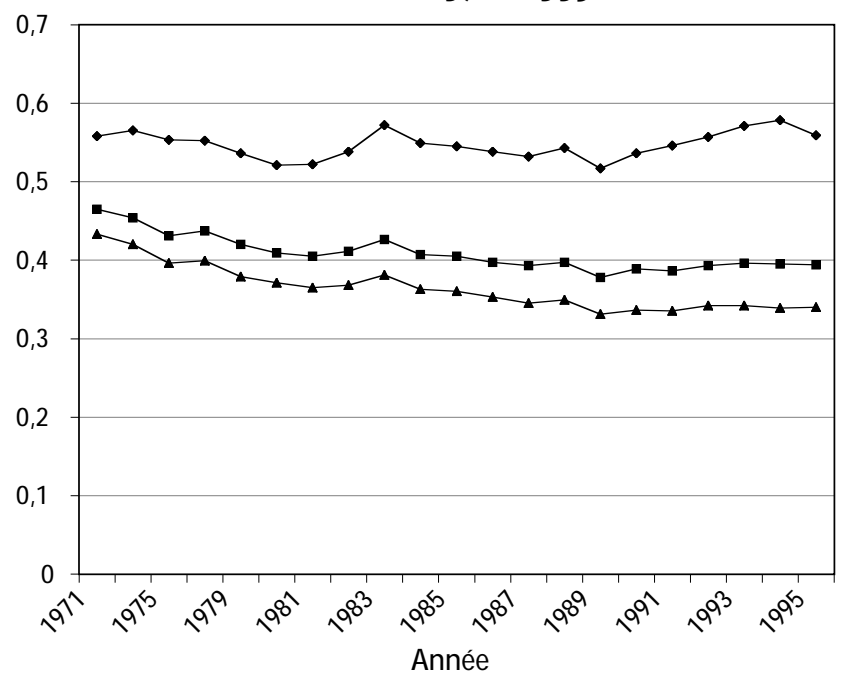

$\rightarrow$ Revenu après impôt $\rightarrow$ Revenu monétaire total $\rightarrow$ - Revenu avant transfert 
figure 6

Coefficients de GINI selon différents concepts de revenu pour les familles, Canada, 1971 à 1995

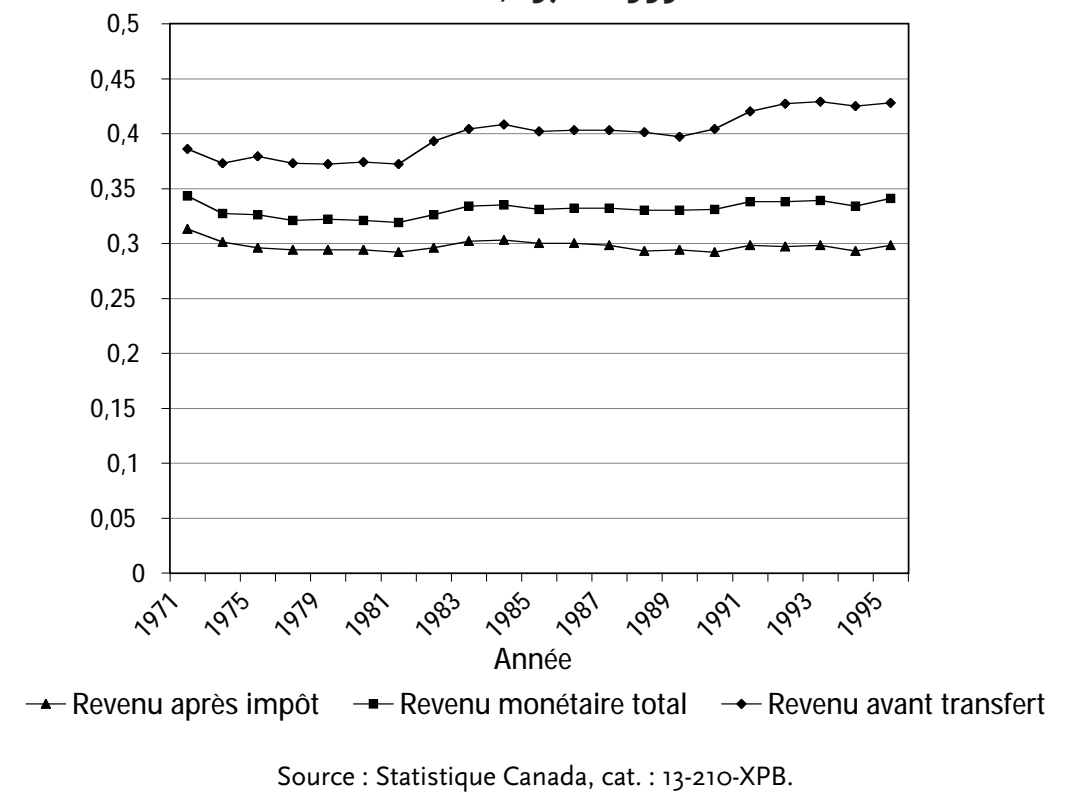

L'impôt a eu comme effet de faire diminuer le taux de faible revenu de $4 \%$, et ce, de manière constante depuis 1980 (Statistique Canada, cat. : 13-592-xpb ; 13-551-xpb). Cequi reflète la contribution des politiques de la fiscalité canadienneà l'amélioration de la situation des couches de la population les plus défavorisées. Cette situation se voit confirmée pour la population dans son ensemble.

En utilisant le coefficient de gin i, nous pouvons remarquer que l'inégalité entre tous les groupes derevenu, les personnes seules et les familles, a augmenté de 1971à 1995, maissi I'on tient compte des impôts et des transferts de paiements, la tendances'est inversée (Statistique Canada, cat. : 13-210-xpb).

De plus, les chiffres sur la mobilité sociale des personnes à faible revenu reflètent une situation assez semblable à celle de tout le monde. Une étude de Noreau et al. (1997) a révélé qu'entre 1993 et 1994, il y avait un taux de roulement de $25 \%$ dans la population de faible revenu selon l'indice du seuil de faible revenu, après impôt. 1,2 millions de Canadiens au-dessus du seuil de faible revenu en 1993 se sont glissés en dessous l'année suivante tandis que 846000 Canadiens en dessous du seuil en 1993 ont grimpéau-dessus en 1994. Pour la population canadienne dans son ensemble, un tiers des gens environ ont changé de quintile de revenu entre 1993 et 1994. Des 5,4 millions de Canadiens dans le quintile le plus pauvre des groupes de revenu en 1993, 29\% ont changé de place en 1994. La mobilité descendante du quintile privilégié était presque aussi importante - de l'ordre de $22 \%$. Une étude de $M$ arie D rolet et René M orisette (1999) portant sur une plus longue période, celle de 1993 à 1996, a confirméce tableau. Environ $80 \%$ des Canadiens ont vécu dans unefamillequi n'a connu aucune période 
de faible revenu entre 1993 et 1996 et seulement environ 5\% des Canadiens ont vécu dans une famille à faible revenu pendant les quatre années consécutives.

La composition des personnes à faible revenu a aussi changé avec le temps. C'est la situation des personnes âgées de 65 ans et plus qui a connu l'amélioration la plus significative. En 1980, un peu plus d'un tiers de cette catégorie d'âge était dans une situation précaire selon la mesure du seuil de faible revenu, une proportion deux fois plus élevée que celle dans la population en général. Seize ans plus tard, le pourcentage a diminué à un peu plus de $20 \%$, cequi représente une proportion à peine plus grande que celle pour la population dans son ensemble. Aujourd'hui ce sont plutôt les jeunes qui gonflent les rangs des personnes à faible revenu, notamment les enfants en bas de six ans et les jeunes âgés entre 16 et 24 ans. Cette situation s'expliqued'ailleurs par lefait que les jeunes parents ont tendance à avoir des jeunes enfants et qu'une plus grande proportion de jeunes parents, tout récemment arrivés sur le marché de l'emploi, gagnent des salaires relativement modestes. M ais il semble qu'il y a en plus un effet de génération, car les jeunes d'aujourd'hui, à un âge comparable, se trouvent dans une situation moins avantageuse que leurs parents : letaux de faible revenu dans les familles dont le chef a moins de 24 ans a grimpé de 20,4\% en 1980 à 42,1\% en 1996.

D'ailleurs, dans le contexte actuel, la contribution du statut matrimonial, ou de la composition familiale, à la pauvreté n'est pas négligeable. Nul besoin de souligner le désavantage économique associéà la monoparentalité. Cependant, certains aspects de cette situation en disent long sur la nature de la pauvreté contemporaine. D'abord, une modification dans la composition familiale affecte les chances des gens de tomber sous le seuil de faible revenu ou d'en sortir. Selon l'étude de Noreau et al. (1997), des per-

figure 7

Proportion de personnes à faible revenu selon l'âge

(SFR base 1992), 1980 à 1996.

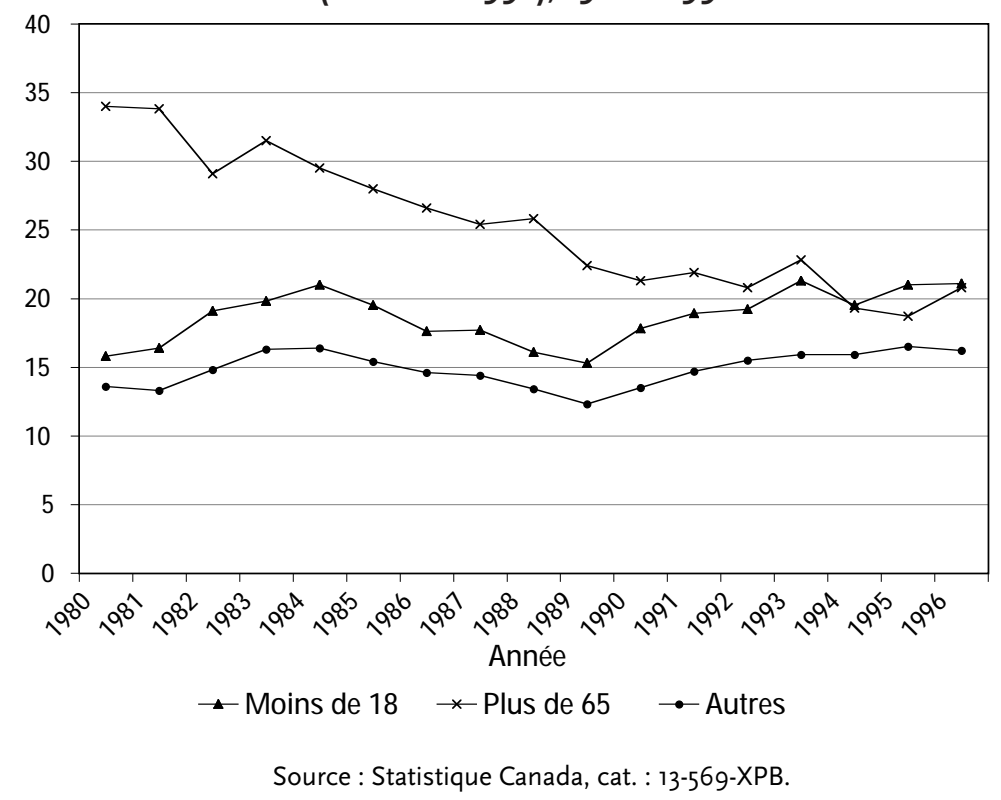


figure 8

Proportion de personnes à faible revenu selon l'âge du chef de famille, 1980 à 1996 (SFR 1992)

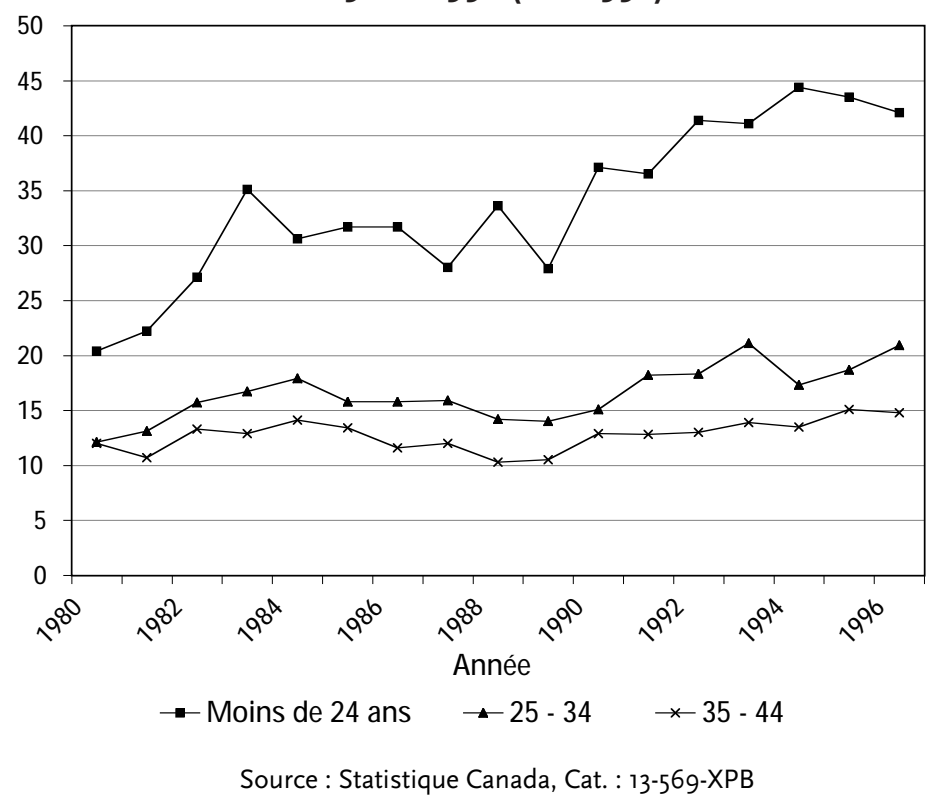

sonnes qui ont connu une telle modification, $17 \%$ ont connu un changement dans leur statut de revenu, un chiffre considérablement plus élevé que celui de $8 \%$ pour la population en général. 41\% de celles qui ont glissé sous le seuil de faible revenu en 1994 ont connu un changement dans la composition de leur famille, tout comme $28 \%$ de celles qui ont grimpéau-dessus du seuil. De plus, deux tiers des membres de familles à faible revenu ayant formé une alliance en sont sortis l'année suivante. Parmi les familles monoparentales dirigées par une femme, un tiers de celles qui ont grimpéau-dessus du seuil en 1994 ont forméune alliance, tandis qu'un quart de celles au-dessus du seuil en 1993 qui se sont séparées de leurs conjoints ont glissé en dessous du seuil. En d'autres mots, la séparation des conjoints aide rarement à sortir de la pauvreté. Chose significative cependant, deux tiers des femmes monoparentales pauvres qui ont réussi à sortir de la pauvreté l'ont fait sans contracter une nouvelle alliance. Dernier aspect à souligner, lorsqu'on examine le revenu déficitaire des personnes à faible revenu - le revenu exigé pour qu'une famille ou un individu atteigne le seuil de faible revenu - , on note que la situation pour toutes les familles et personnes seules est demeurée assez stable entre 1980 et 1996, mais elle s'est renversée pour les familles biparentales et monoparentales. La situation des familles avec deux parents s'est détériorée de 320 , tandis que celle des familles monoparentales s'est améliorée de 639 \$.

II est à noter que ces résultats sur la contribution du statut matrimonial au faible revenu ont été confirmés par une étude récente (Picot, et al., 1999). Pour ces auteurs, «le mariage avait un effet spectaculaire» (ibid., p. 12) sur la proportion des enfants qui sont sortis du faible revenu. $99 \%$ des enfants dont leparent seul s'est marié sont sortis de leur 
figure 9

Revenu déficitaire en dollars constants (1996) pour certains genres d'unités familiales à faible revenu, 1980 à 1996.

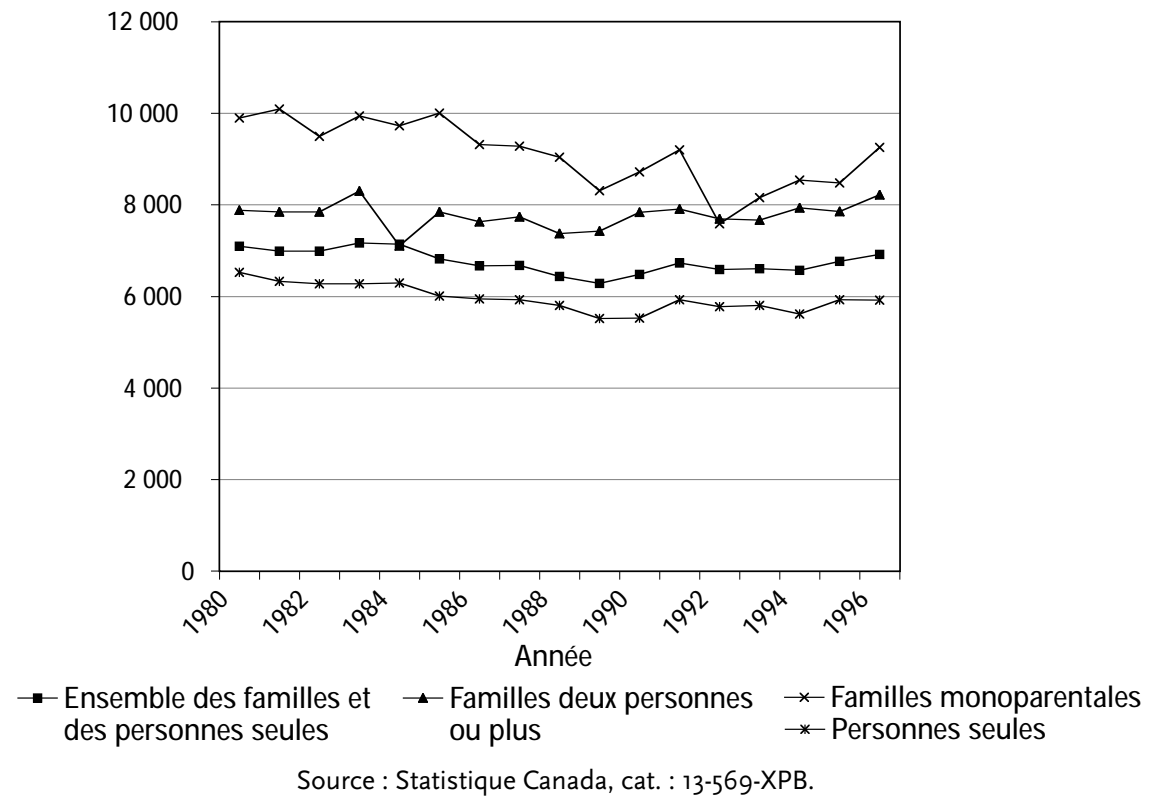

situation de faible revenu comparé à $21 \%$ de ceux appartenant à des familles où il n'y avait pas eu de nouvelle alliance entre 1993 et 1994. M ais les auteurs soulignent également que cette situation touche très peu de gens - seulement $6,7 \%$ des enfants appartenaient à des familles où un mariage a eu lieu. C'est pourquoi une amélioration dans leurs gains hebdomadaires représente un facteur aussi important que la composition familiale dans l'incidence des chances des pauvres de sortir de la pauvreté.

\section{la portée des statistiques}

Bon nombre de sociologues diraient que ces statistiques ne prouvent rien. D'autres diraient qu'on peut faire dire n'importe quoi aux statistiques. Pourtant, les statistiques révèlent seulement ce dont elles parlent. Et ce dont il s'agit ici est la question suivante : quelle est la meilleure description et, par conséquent, explication de la pauvreté contemporaine? Si nous avons cité ces statistiques plus haut, c'est parce qu'elles nous semblent souligner les aspects saillants de la pauvreté contemporaine: les pauvres ne constituent pas un groupe homogène, pris dans une spirale de reproduction dont la cause est attribuable à leur exclusion systématique du marché de travail et tutti quanti et dont la situation empire à cause du démantèlement de l'État-providence, fruit du néolibéralisme et de la globalisation. Au contraire, les pauvres sont mobiles, hautement différenciés, et leur situation s'explique par plusieurs facteurs. Parmi ces facteurs, certains sont la conséquence de cycles économiques, certains résultent des politiques gouvernementales et d'autres encore sont les résultats de choix individuels. À titre d'exemple : si la composition familiale est un facteur important associé à la pauvreté, 
il se peut quel'aspect le plus remarquable de cette association soit le fait que deux tiers des femmes monoparentales qui ont réussi à sortir de la pauvreté l'ont fait sans contracter une nouvelle alliance. M algré le fait que les gens savent que la séparation et le divorce peuvent conduire directement à la pauvreté, ils continuent de faire ces choix. Et ils les font parce que les arrangements sociaux sont tels qu'ils peuvent se les payer. Ils savent que, statistiquement, ils ne sont pas condamnés à vie à être pauvres. Le gouvernement est sensibilisé au sort des familles monoparentales et a pris des initiatives, surtout fiscales, pour en tenir compte. La preuve : la situation des familles monoparentales pauvres s'est améliorée comparativement à celle des familles biparentales. Et si le gouvernement répond à cette situation, c'est parce que l'évolution de la société est telle que les choix amoureux sont détachés des considérations pécuniaires. L'entrée massive des femmes sur le marché du travail souligne cet état et rebondit sur le domaine amoureux en libérant l'amour de toute considération autre que l'apport de la gratification des besoins et des désirs intimes (Laurin-Frenette, 1981; Luhmann, 1986). Que les couples s'écroulent sous ce poids est autre chose, mais la séparation des sphères reflètel'autonomisation grandissante, voire l'autoréférentialité, de chaque sous-système de la société. Avec, comme corollaire, que chaque domaine de la vie est traversé par des effets non intentionnés des décisions prises dans d'autres domaines. Et certaines de ces décisions ne sont pas assujetties à l'intervention étatique. On peut imaginer un scé nario où le gouvernement interdirait aux couples avec enfants de divorcer jusqu'à ce que les enfants atteignent l'âge de dix ans. À en croire nos chiffres, cela diminuerait les taux de pauvreté de beaucoup, mais une telle politique est peu envisageable. Comme l'écrivait Luhmann (1990), le pouvoir est un média symbolique inadéquat pour modifier le comportement individuel et il est inadéquat parce qu'un tel décret irait à I'encontre des tendances structurelles de notre société vers I'accroissement de la liberté des individus dans tous les domaines, tendances appuyées par le processus de différenciation interne qui caractérise la modernité par excellence.

En d'autres termes, nos sociétés sont caractérisées par un processus d'inclusion grandissante. Deplus en plus de personnes ont accès à de plus en plus de ressources, autant matérielles que symboliques, dans de plus en plus de domaines. C'est ce que traduisent l'individualisation grandissante de nos sociétés, leur démocratisation accrue, la prolifération de groupes d'intérêts de toutes sortes qui revendiquent reconnaissance et appui. Les pauvres, et les différentes catégories de pauvres, n'en sont qu'un exemple parmi d'autres. Et de ce point de vue, les pauvres ne sont guère différents de leurs homologues dans l'ensemble du système de stratification, où les places sont distribuées sur la base d'un mariage entre le talent et la chance (Schecter, 1993). II faut comprendre par le talent non seulement l'intelligence scolaire mais la panoplie des habiletés individuelles tels l'entregent, la débrouillardise, la séduction, la ténacité, etc., tout comme il faut comprendre par la chance autant lehasard météorologique, l'accident de naissance, que les cycles économiques, etc. (Jencks, 1972). Un tel système de stratification n'est plus divisé en classes sociales identifiables. Il est plutôt caractérisé par un haut degré de mobilité ascendante et descendante, par plusieurs voies de réussite et 
par plusieurs formes de récompense qui ne sont pas nécessairement cumulatives. Certes, il demeure des inégal ités, mais ce ne sont plus des inégalités de classes, tout comme il demeure des pauvres, mais ce ne sont plus les pauvres d'antan. Nos pauvres sont plutôt mobiles, revendicatifs, politiquement organi sés. Certains sont mêmetrès instruits, car les étudiants universitaires grossissent pendant quelques années de leur vie les rangs des pauvres (Drolet et M orissette, 1999). Et en réponse à leurs revendications, le gouvernement agit. Une preuve parmi d'autres : le déclin dans les taux de pauvreté, presque universel dans les pays occidentaux depuis les années soixante-dix, est principalement le résultat des politiques qui visai ent la situation des personnes âgées. Les statistiques le confirment et, en le confirmant, soulèvent des interrogations sur les thèses du dé mantèlement de l'État-providence. (Rosanvallon, 1995 et 1984) Le fait que le gouvernement maintenant cible davantage les groupes particuliers lors de ces programmes sociaux peut n'être que sa réponse au caractère de plus en plus multiforme des groupes, dont les pauvres, qui sollicitent son aide. Une situation que les chiffres sur les pauvres, les jeunes et les personnes âgées tendent à confirmer.

M ais cela ne doit pas cacher le processus d'inclusion à l'œuvre. Comme l'écrivait Luhmann (1990), dans une société qui fonctionne sur la base de la différenciation grandissante, même les pauvres doivent être solvables. On peut ajouter que c'est précisément cettenécessitéstructurelle qui donneaux discours deressentiment envers les «fraudeurs du bien-être» leur caractère loufoqueet obscène. Pourtant, ce regain de malthusianisme rencontre son homologue de l'autre côté de l'échiquier politique lorsque les champions de la critique du néolibéralismer refusent d'imaginer autrechoseque la descente aux enfers comme destin qui se dessine à I'horizon. En effet, il semble aujourd'hui que la gauche et la droite convergent inconsciemment dans leur commun attachement à des autodescriptions obsolètes de la société. La discussion incessante autour de la pauvreté infantile en témoigne. Pour la gauche, elle est signe de l'indifférence criante d'une sociétéqui produit de plus en plus d'exclus. Pour la droite, elle confirmel'irresponsabilité morale de parents qui ont tout pour mieux faire.

\section{la pauvreté contemporaine et la théorie sociologique}

Les données canadiennes semblent converger vers la même conclusion que celle des chercheurs qui doutent de la validité des thèses de l'underclass et de l'exclusion. N ous comprenons que cette mise en question soit difficileà endosser lorsque les scènes de la vie urbaine quotidienne nous offrent un tableau visible de la misère : les quémandeurs devant les portes d'un métro ou d'une banque, les désinstitutionnalisés criant dans les rues et dans les autobus, les jeunes offrant de nettoyer la vitre d'une voiture arrêtée momentanément au feu rouge contre une pitance. M ais les statistiques racontent elles aussi leurs histoires, nous offrant un tableau de l'évolution de la pauvreté qui exige qu'on repense les concepts par lesquels nous analysons ce phénomène. Et c'est ainsi que nous suggérons que la nouvelle forme de la pauvreté contemporaine nous convie à modifier la manière dont nous analysons à la fois le système de stratification et la société dans son ensemble. 
Les pauvres ne sont pas systématiquement pauvres. Ils ne se reproduisent pas génération après génération. Les raisons de la pauvreté sont multiples. Elles changent avec les années et avec les différentes catégories de pauvres. Les sources de la pauvreté ne sont pas toutes d'ordre social et les remèdes à la pauvreténe sont pas tous à la portée d'une intervention efficace de la part des autorités publiques. Parfois, I'argent est utile pour améliorer la situation de certaines populations de pauvres, mais dans d'autres circonstances, cela risque ne pas s'avérer être le cas. Pensons, par exemple, à ceux et à celles dont la pauvreté est liée à des déficiences mentales ou personnelles quelconques. Dans la mesure cependant où ce facteur est une «explication » de la pauvreté, il dirige notre attention sur les facteurs qui entrent en jeu dans la détermination des places dans la société en général. Et si, parmi ces facteurs, les talents individuels, définis dans un sens large pour comprendre toutes sortes d'habiletés psychologiques, jouent un rôle de plus en plus important, n'est-ce pas parce que la société elle-même leur accorde une place importante? Pourtant, dans quel type de société ces talents peuvent-ils avoir un effet, si ce n'est pas dans une société de plus en plus mobile, où les chances et les avenues de réussite sont de plus en plus multiformes? Dela même manière, dans quel type de société la chance peut-elle avoir une résonance sociétale, si ce n'est pas dans une société de plus en plus ouverte, où les places ne sont plus fixées selon la classe, sans parler de l'ordre?

La nouvelle forme que revêt la pauvreté contemporaine reflète ce que Pakulski et Waters (1996) ont décrit comme la mort des classes. Mais elle reflète également l'interprétation de la société contemporaine que Luhmann (1993 et 1995) nous a décrite : une société fonctionnellement différenciée qui nous présente des paradoxes là où I'analyse classique de la modernité voyait des contradictions. La société moderne ne se présente plus sous la forme d'une classe contre une autre, d'un lieu central qui domine la société dans un sens marxiste ou weberien. Elle est mieux saisie sous la forme de ses multiples systèmes sociaux, chacun fonctionnant sur la base du média symbolique général d'échange qui lui est spécifique et qui circule selon les codes opératoires dans son système. Chaque sphère peut pénétrer l'autre, mais seulement dans la mesure où elle traduit ses demandes sous une forme adéquate au système qu'elle veut influencer. Pour donner un exemple, l'argent ne peut pas acheter une élection. II ne peut pas non plus acheter l'amour, tout comme le pouvoir ne peut pas l'acheter. II peut, bien sûr, aider, mais il ne peut pas garantir la réussite.

Très souvent d'ailleurs, la tentative de répondreaux demandes qui lui sont adressées met le système en question en difficulté. II arrive de plus en plus fréquemment que les décisions prises pour satisfaire les demandes entraînent des conséquences imprévues qui, à leur tour, génèrent de nouvelles demandes et de nouvelles difficultés. Et c'est d'autant plus le cas que chaque système social est caractérisé par l'inclusion grandissante de la population dans sa sphère d'activité. Historiquement, la modernité se caractérise non seulement par l'autonomisation des sphères sociétales - la séparation de l'Église et del'État, pour prendre l'exemple le plus notoire - mais aussi par la démocratisation de celles-ci : lesuffrage universel pour le systèmepolitique, l'enrichissement des ouvriers 
pour le système économique, la participation de tous dans les déboires de l'amour romantique indépendamment de leur statut de classe ou de citoyenneté, etc. C'est dans ce sens que l'exclusion n'est plus un concept théoriquement adéquat pour rendre compte de la nature de la pauvreté contemporaine, tout comme la classe n'est pas un concept adéquat pour saisir la nature des inégalités qui sont les nôtres.

Une des caractéristiques d'une société modernequi fonctionne telle que décritepar Luhmann est le paradoxe. On a des riches et on a des pauvres et, dans un certains sens, on a des pauvres parce qu'on a des riches, mais pas comme $M$ arx l'entendait. On pourrait même dire qu'on a les pauvres de nos riches, car certes ils sont des pauvres d'une société démocratique et abondante. Et faceà eux, on a des attitudes qui sont à des annéeslumières du début de la modernité où l'on pendait les pauvres pour vagabondage, mais on se trouve également devant un autre paradoxe de la modernitétardive : celui où l'on ne peut pas toujours tout régler, chaque décision entraînant des conséquences qu'on ne peut pas prévoir et chaque évolution de la société rendant la maîtrise d'une situation de plus en plus complexe et difficile. Il est évident qu'on ne peut plus selivrer aux illusions utopiques de la transformation totale de la société, car elle n'est pas elle-même saisissable dans sa totalité. Régler la question de la pauvreté participeau même dilemme. Cequi ne veut pas dire que nous ne pourrions rien faire. II veut dire simplement que nous ferons mieux de tout faire pour identifier les différents paramètres de la situation, tout en comprenant que l'analyse ne comportera pas d'emblée une prescription de remède. Parfois même, nous devrions méditer longtemps avant d'agir, quitte à renoncer à certaines interventions.

Pour prendre un exemple, l'étude de Picot et al. (1999) a soulignéla contribution de l'éducation et surtout de l'éducation post-secondaire à l'amélioration des chances de sortir de la pauvreté. M ais on ne peut ni obliger les gens à fréquenter l'école au-delà d'un certain âge - on a déjà du mal à faire en sorte que les jeunes y restent et apprennent - ni introduire des réformes de programmes postsecondaires pour attirer de nouvelles clientèles sans vider davantage les institutions du haut savoir de leur fonction intellectuelle. Làil nes'agit plus simplement d'une question éthique mais de la difficulté de résoudre une question sociale dans un domaine sans affecter un autre lieu institutionnel dans une direction qu'on pourrait regretter par la suite. Et c'est encore davantage le cas lorsqu'on tient compte des statistiques montrant que les facteurs qui déterminent le niveau de scolarité obtenu sont loin d'être ceux qu'on pense et encore moins malléables par les variables institutionnelles (Jencks et al., 1972).

Plus on avance dans la modernité, semble-t-il, plus on découvre le sens des vérités de la tradition. Quant aux pauvres, il ne s'agit pas seulement de ce qu'en a dit l'Évangile : les pauvres seront toujours avec nous. II s'agit également de ce quel'Évangilea dit de nous tous : pardonnez-leur, Seigneur, ils ne savent pas ce qu'ils font. Si la sociologie peut nous rappeler les vertus autant de la prudence que de la charité, peut-être l'avenir sera autre chose que la reproduction du passé. î 


\section{résumé}

Depuis le début des années 1980, les phénomènes liés à la pauvreté et à la précarité sociale sont généralement abordés à l'aide du concept d'exclusion sociale, concept popularisé en Europe et développé particulièrement en France par des auteurs comme Robert Castel et Serge Paugam. Peut-être ce concept concorde-t-il à la situation française, mais la réalité canadienne semble bien différente. Les différentes statistiques liées à la pauvreté, à l'inégalité et à la mobilité sociale au Canada ne permettent pas de corroborer l'hypothèse de l'exclusion. Selon nous, le phénomène inverse, c'est-à-dire une inclusion grandissante, dans le sens que suggère la sociologie de Niklas Luhmann, est à l'oeuvre. Dans ce texte nous confrontons les deux hypothèses avec les données statistiques canadiennes afin de démontrer que la tendance à long terme de la société contemporaine s'oriente vers l'inclusion.

\section{summary}

Since the 1980s, the phenomena linked to poverty and social precarity have generally been addressed using the concept of exclusion, a concept popularized in Europe and developed particularly in France by authors such as Robert Castel and Serge Paugam. While this concept might apply to the French case, Canadian reality seems quite different. Various statistics relating to poverty, inequality and social mobility in Canada do not corroborate the exclusion hypothesis. For us, the opposite phenomenon is at work, namely increasing inclusion, in the sense suggested by the sociology of Niklas Luhmann. In this text we shall confront the two hypotheses with Canadian statistical data in order to show that the long term tendency of contemporary society points toward inclusion.

\section{resumen}

Desde el comienzo de los años 1980, los fenómenos ligados a la pobreza y a la precaridad social son generalmente abordados utilizando el concepto de exclusión social, concepto popularizado en Europa y desarrollado particularmente en Francia por autores como Robert Castel y Serge Paugam. Es posible que este concepto concuerde con la situación francesa, pero la realidad canadiense parece bien diferente. Las distintas estadísticas ligadas a la pobreza, a la desigualdad y a la mobilidad social en Canadá no permiten corroborar la hipótesis de la exclusión. Al contrario, nosotros consideramos que es el fenómeno inverso que se produce, es decir una inclusión creciente en el sentido que lo sugiere la sociología de Niklas Luhman. En este texto, nosotros confrontamos las dos hipótesis con los datos estadísticos canadienses afin de demostrar que la tendencia a largo plazo de la sociedad contemporánea se orienta hacia la inclusión.

\section{bibliographie}

Aul et ta, Ken (1982), The Underclass, New York, Vintage Books.

Cast el , Robert (1995a), Les métamorphoses dela question sociale, Paris, Fayard.

Cast el , Robert (1995b), «Le piège del'exclusion», Lien social et politique, no 34, p. 13-22.

Cast el, Robert (1994), «La dynamique des processus de marginalisation : de la vulnérabilité à la désaffiliation », Les cahiers de recherche sociologique, $\mathrm{n} 022$, p. 11-27.

Cast el , Robert (1992), «Del'indigence à l'exclusion, la désaffiliation », Faceà l'exclusion : le modèle français, sous la dir. D onzel ot , Jacques, p. 137-168, Paris, Éditions Esprit. 
Cl ar k, T. N. et S. M. Lipset (1990), «Are Social Classes Dying?», International Sociology, no 6, p. 397-410. Conseil national du bien-êtresocial (1998), Profil dela pauvreté, 1996, Ottawa, M inistre des Travaux publics et services gouvernementaux Canada, printemps.

Do nzel ot , Jacques (dir.) (1992), Faceà l'exclusion : le modèle français, Paris, Éditions Esprit.

D r ol et, M arie et RenéM or iset t e (1999), Dans quellemesure les Canadiens sont-ils exposés au fai ble revenu? Ottawa, Statistique Canada, mars.

Fassin, Didier (1996), «Exclusion, underclass, marginalidad : figures contemporaines de la pauvreté en France, aux États-Unis et en Amérique latine», Revue française de sociologie, no xxxvii, p. 37-75.

Fel I egi i, Ivan P. (1998), À propos de la pauvreté et du faible revenu, Statistique Canada, Cat. no 13F0027XIF. Gaut h ier , M adeleine (1995), «L'exclusion, une notion récurrente au Québec mais peu utilisée ailleurs en Amérique du Nord», Revue internationale d'action communautaire, no 34, p. 151-156.

Gaut h ier , M adeleine (sous la dir.) (1987), Les nouveaux visages de la pauvreté, Q uébec, Institut Québécois de Recherche sur la Culture.

Human Resources Development Canada (1998), «The Market Basket M easure - Constructing a N ew Measure of Poverty», Applied Research Bulletin, Ottawa, mars.

Jencks, Christopher (1992), Rethinking Social Policy: Race, Poverty, and the Underclass. Cambridge, MA, $\mathrm{H}$ arvard University Press.

Jencks, Christopher et P. E. Pet er son (eds.) (1991), The Urban Underclass, Washington, The Brookings Institute.

Jencks, Christopher, M. Smit h, H. Ackl and , M. J. Bane, D. Cohen, H. Gint is, B. H eyns, et S. M ichel son (1972), Inequality : A Reassessment of the Effect of Family and Schooling in America, New York, Basic Books.

Kat z, M ichael B. (dir.) (1993), The Underclass D ebate: Views From History. Princeton, N .J. : Princeton University Press.

Langl ois, Simon (1997), «Pauvreté, dépendance sociale et inégalités : la situation au Q uébec», in R. Côt é (ed.), Québec 1997, p. 39-63. M ontréal, Le Devoir et Fides.

Langl ois, Simon (sous la dir.) (1991), La société québécoise en tendances, 1960-1990, Québec, Institut Québécois de Recherche sur la Culture.

Langl ois, Simon (1987), «Les seuils de pauvreté» in Gaut h ier , M . (sous la dir.), Les nouveaux visages de la pauvreté, Québec, Institut Québécois de Recherche sur la Culture.

Lau r in-Fr enet t e, Nicole (1981), «Féminisme et anarchisme : quelques éléments théoriques et historiques pour une analyse de la relation entre le M ouvement des femmes et l'Etat», Femmes et politiques, sous la dir. Cohen, Yolande, p. 147-191, M ontréal, Le jour, éditeur.

Lion, A. et P. M acl o uf (1982), L'insécurité sociale, Paris, Éditions ouvrières.

Lu h mann, Niklas (1995), Social Systems, Stanford, Stanford University Press.

Luh mann, Niklas (1993), Risk: A Sociological Theory, New York, A. DeGruyter.

Luh mann, Niklas (1990), Political Theory in the WelfareState, Berlin-N ew York, Walter de Gruyter.

Luh mann, Niklas (1986), Love as Passion: On the Codification of Intimacy, Cambridge, Harvard University Press. (En français : Amour comme passion, 1990, Paris, Aubier.)

M or iset t e, René, C. Bér ubé (1996), Aspects longitudinaux de l'inégalité des revenus au Canada, Ottawa, Statistique Canada, étude analytique $n-94$.

No r eau, Nathalie, M. Webber , P. Gil I is et A. H al e (1997), Traverser le seuil de faible revenu, Ottawa, Statistique Canada.

Pakul ski, J. et M. Wat er s (1996), The Death of Class, London, Sage.

Paqu et, Bernard (1996), Stratification sociale, exclusion et underclass, Département desociologie, Université du Q uébec à M ontréal, miméo.

Paqu et, Bernard (1994), «Exclusion et inégalités dans la société contemporaine : éléments d'interprétation », M émoire de maîtrise, Québec, Université Laval.

Par sons, Talcott (1977), Social Systems and the Evolution of Action Theory, N ew York, The Free Press.

Pau gam, Serge (sous la dir.) (1996), L'exclusion : I'état des savoirs, Paris, Éditions La Découverte. 
Pau ga m, Serge (1991), La disqualification sociale : essai sur la nouvelle pauvreté, Paris, puf .

Pau gam, Serge, J.-P. Zoyem et J.-M . Ch ar bonnel (sous la dir.) (1993), Précarité et risque d'exclusion en France, Paris, Centre d'étude des revenus et des coûts (C.E.R.C.), La documentation française.

Picot, G., M . Zybl o ck et W. Pyper (1999), Qu'est-cequi expliquele mouvement des enfants versla situation defaible revenu et hors de celle ci, les changements de situation sur lemarchédu travail ou le mariage et le divorce? Ottawa, Statistique Canada, avril.

Rosanval I on, Pierre (1995), La nouvelle question sociale : repenser I'État-providence, Paris, Seuil.

Rosanval I on, Pierre (1984), La crise de l'état-providence, Paris, Seuil.

Ross, David P., E. R. Shillington et Clarence Lockhead (1994), The Canadian Fact Book on Poverty, Ottawa, Canadian Council on Social Development.

Sa r l 0, Christopher A. ( 1996), Poverty in Canada ( $2^{\text {nd }}$ Edition), Vancouver, The Fraser Institute.

Schect er , Stephen (1998), «De la limite et de la richesse dans la société québécoise contemporaine». Jewsi ewicki B. et J. Létourneau (dir.), Identités en mutation : socialités en germination, Québec, Septentrion, p. 185-95.

Sch ect er , Stephen (1995), «Luhmann et lepolitique: au delà del'incroyable», Société, M ontréal, no 14, p. 35 à 66 .

Sch ect er , Stephen (1993), «De la stratification sociale dans la société postmoderne», Société, no 11, p. 5794.

Sch ect er , Stephen et Bernard Paquet (1999), «Contested Approaches in the Study of Poverty: The Canadian Case and the Argument for Inclusion», Current Sociology, vol. 47, no 3, p. 43-64.

Sch napper , Dominique (1981), L'épreuve du chômage, Paris, Gallimard.

Statistics Canada (1996a), Low IncomeAfter Tax, cat. 13-592-XPB.

Statistics Canada (1998), Low Income M easure, cat. 13-582-XPB.

Statistics Canada (1996b), Low Income Persons, cat. 13-569-X PB.

Statistics Canada (1997), Low Income Cut-offs, cat. 13-551-X PB.

Statistics Canada (1996), Income Distributions by Size in Canada, cat. 13-207 XPB.

Statistics Canada (1998), can sim Database.

Statistics Canada (1995), Income After Tax, Distributions by Size in Canada, cat 13-210-XPB.

W ebber, Maryanne (1998), La mesure du faible revenu et de la pauvreté au Canada : une mise à jour, Statistique Canada, cat 75F 0002M .

W il son, William-Julius (ed.) (1993), The Ghetto Underclass: Social Science Perspectives. N ewbury Park, CA, Sage.

W il son, William-Julius (1987), The Truly Disadvantaged: The Inner City, The Underclass, and Social Policy, Chicago, University of Chicago Press.

W ol f son, M. C. et B. B. M ur phy (1998), «N ew views on inequality trends in Canada and the United States», M onthly Labor Review, U.S. Department of Labour, p.3-23, avril.

Zel I ey, E.-Walton (1995), «Isthe Underclass Really a Class?», Journal of Sociology and Social Welfare, vol. 22, no 1, p. 75-85. 